\title{
Optical Properties of Micro and Nanostructured Bioinspired Materials
}

\author{
Abdessitir Deraoui ${ }^{*}$, Kamel Mallat, Alain Cornet, Pierre Defrance \\ Institute of Condensed Matter and Nanoscience (IMCN)/Nanophysics (NAPS), Université Catholique de Louvain Belgium
}

\begin{abstract}
The nature shows us a great many situations where the optics plays a crucial part; this is particularly the case for birds and insects whose color is usually associated with their behavior as a factor of recognition. This variation of coloration depends on the illumination and depending on the direction of observation: it is the phenomenon of iridescence. The material forming for example the elytra of beetles is the chitin and its refractive index is close to that of ordinary glass. The SEM observation shows that the material is in the form of multilayer separated by layers of air. These layers disturb the refractive index of chitin and cause multiple reflections which interfere and explain the phenomenon of iridescence. The models clearly confirm this interpretation [1], but they also show that it is possible to produce structured surfaces in the laboratory with properties similar to natural structures. By using design ideas from nature we are able to work towards the development of applications in a range of different technologies. This paper aims to develop methods for producing samples based on natural structures where optical properties can be interesting. We will review typical bio-inspired photonic structures and focus on the biomimetic fabrications. These samples will be characterized by various methods in order to understand its properties.
\end{abstract}

Keywords Optical Properties, Iridescence, Multilayer, Photolithography, Anisotropic Wet Etching, Structured Surfaces, Scatterometry

\section{Introduction}

The physical origin of the coloration of insects has been suspected very early $[2,3]$ and directly confirmed by electron microscopy as soon as this technique was made available [4]. Since then, one- [5], two- [6] and even three-dimensional [7] photonic crystals have been shown to be responsible for the coloration of many living organisms [8].

Structural colours created by multilayer are widely used in the animal kingdom for display and/or camouflage [9-11]. There are many mechanisms by which structural colours can be produced. All depend directly or indirectly on the fact that a particular piece of material scatters or refracts different wavelengths of light to different degrees. This property of the material can be expressed in terms of its index of refraction, $n$, a measure of the degree to which a given wavelength of light entering the material is "retarded" or slowed down. Structural colours described so far in biological systems fall into two general classes, scattering and interference.

The general category of interference includes those situations in which the rays of a beam of white light are

* Corresponding author:

abdessitir.deraoui@uclouvain.be (Abdessitir Deraoui)

Published online at http://journal.sapub.org/optics

Copyright (C) 2014 Scientific \& Academic Publishing. All Rights Reserved temporarily separated and then brought back together in such a manner that some have traveled a longer path than others. Depending on the geometry, when the rays recombine, certain wavelengths are in phase and reinforced ("constructive interference"); these will shine with particular brilliance. Others are out of phase and cancel each other ("destructive interference"). The results are the shimmering colors we call "iridescent." There are many ways of producing iridescence; this article considers only those of known importance in insects.

It is now well known that submicron structures are responsible for the structural colour through light interference in layered or lattice structures and also Tyndall scattering [12].

The physical structure and surface chemistry of these insects provides surprising properties that could offer a variety of applications ranging from photonic security tags to self cleaning surfaces and protective clothing and to industrial sensors.

In this work, we created structures by etching periodic pyramid on surfaces of a flat sample of silicon and coated it with an alternating multilayer of the materials. The optical properties of the bio-inspired structures are also investigated.

The combination of the nanofabrication technique, useful design methodologies inspired by biological systems would surely have profound impacts on our society.

Subsequently, a theoretical model has been successfully proposed to explain the observed extraordinary reflective 
properties.

\section{Material and Methods}

We wanted to fabricate a sample as shown in Fig.1 below. A pyramid with a base opening of $\mathrm{D}=100 \mu \mathrm{m}$ and $\mathrm{h}=50 \mu \mathrm{m}$ of thickness (see Fig.1). We describe in detail in this paper the micro and nanofabrication steps made in the cleanrooms.

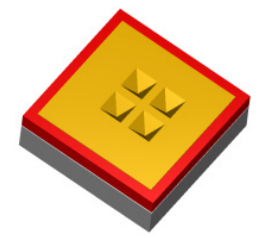

Figure 1. Design of the pyramid system

\subsection{Photolithography}

Photolithography is the standard process to transfer a pattern, which has been designed with computer-aidedengineering (CAE) software packages, on to a certain material. The process sequence is illustrated in Fig.2 A mask with the desired pattern is created thanks to Cadence Virtuoso Layout Editor IC 5.0.0.

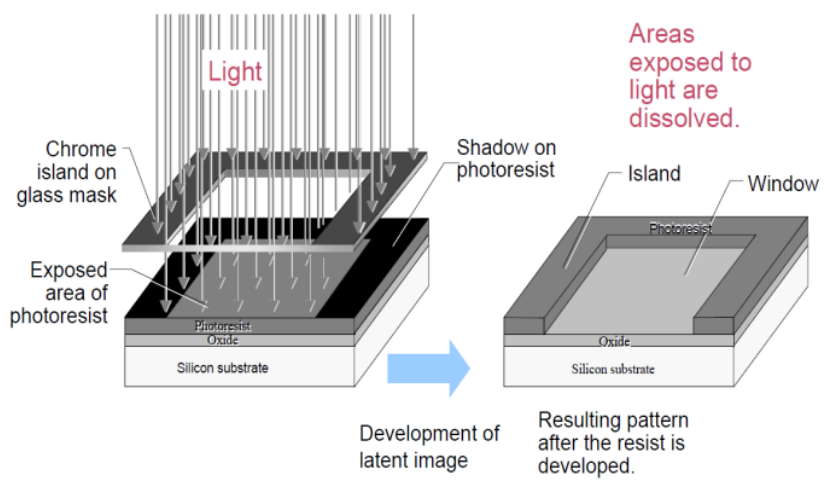

Figure 2. Schematic of a photolithographic process sequence to structure a thinfilm layer

The mask is a glass plate with a patterned opaque layer (typically chromium) on the surface. Electron-beam lithography is used to write the mask pattern from the CAE data. In the photolithographic process, a photoresist layer (photostructurable polymer) is spin-coated on to the material to be patterned.

Next, the photoresist layer is exposed to ultraviolet (UV) light through the mask. This step is done in a mask aligner, in which mask and wafer are aligned with each other before the subsequent exposure step is performed. Depending on the mask aligner generation, mask and substrate are brought in contact or close proximity (contact and proximity printing) or the image of the mask is projected (projection printing) on to the photoresist-coated substrate.

Depending on whether positive or negative photoresist was used, the exposed or the unexposed photoresist areas, respectively, are removed during the resist development process.
The remaining photoresist acts as a protective mask during the subsequent etching process, which transfers the pattern onto the underlying material. Alternatively, the patterned photoresist can be used as a mask for a subsequent ion implantation. After the etching or ion implantation step, the remaining photoresist is removed, and the next layer can be deposited and patterned.

\subsection{Anisotropic Wet Etching}

Liquid etchants etch crystalline materials at different rates depending upon which crystal face is exposed to the etchant. There is a large difference in the etch rate depending on the silicon crystalline plane. In materials such as silicon, this effect can allow for very high anisotropy. Some of the anisotropic wet etching agents for silicon are potassium hydroxide $(\mathrm{KOH})$, ethylenediamine pyrocatechol (EDP), or tetramethylammonium hydroxide (TMAH). Etching a (100) silicon wafer would result in a pyramid shaped etch pit as shown in Fig.3. The etched wall will be flat and angled. The angle to the surface of the wafer is $54.7^{\circ}$.

The relationship between mask dimensions, etch depth and the floor width is given in equation 1 .

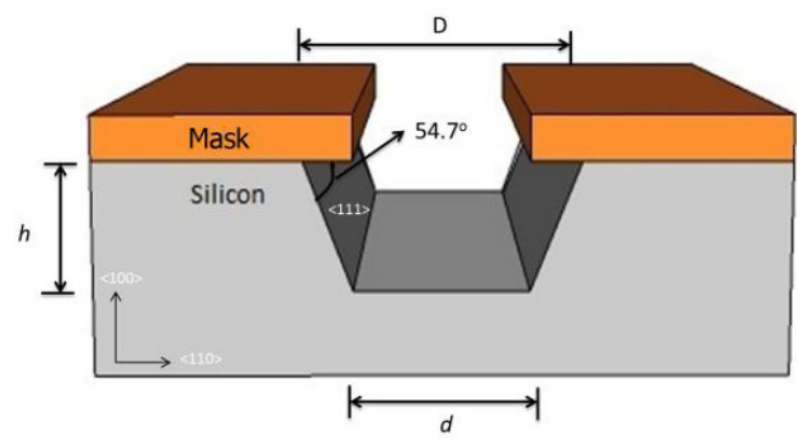

Figure 3. Anisotropic wet etching a (100) silicon wafer

$$
d=D-\frac{2 h}{\tan \left(54.7^{\circ}\right)}
$$

\subsection{The Coating}

In order to produce the precise visual effect observed on 'Chrysochroa vittata', the structure is copied. The chitin plate $(\mathrm{n}=1.56)$ will be replaced by a layer of silicon monoxide $\mathrm{SiO}(\mathrm{n}=1.9)$ and the nickel $\mathrm{Ni}(\mathrm{n}=1.85)$ replaced the air layer. The purpose of choosing nickel is simply to produce a periodic perturbation in order to pen the one-dimensional photonic gaps. The multilayer alternates a silicon monoxide layer $170 \mathrm{~nm}$ thick with $10 \mathrm{~nm}$ layer nickel spacer.

The deposition of $\mathrm{SiO}$ was made by evaporation from an electrically heated (by Joule effect) tantalum crucible, and the coating of nickel was applied by the evaporation of the nickel cathode, impacted by accelerated electrons driven by the static magnetic field of a permanent magnet.

And because we have several facets in our pyramidal sample, what explains that we didn't have the same thickness on all facets. To determine experimentally the effect of 
variation of angles, we deposited the same multilayer on flat substrates placed on oblique supports of $45^{\circ}$ with the rotating plate of the evaporator and we measured the thickness of the coating thanks to Dektak profiler.

\subsection{Morphology Characterization}

A schematic of a coherence scanning interferometer is shown in fig.4 (left). The upper beam splitter directs light from the light source towards the objective lens. The lower beam splitter in the objective lens splits the light into two separate beams. One beam is directed towards the sample and one beam is directed towards an internal reference mirror. The two beams recombine and the recombined light is sent to the detector. Due to the low coherence of the white light source, the optical path length to the sample and the reference must be almost identical, for interference to be observed. Note that coherence is the measure of the average correlation between the value of a wave at any pair of times, separated by a given delay. Temporal coherence tells us how monochromatic a source is. In other words, it characterises how well a wave can interfere with itself at a different time (coherence is discussed in detail in the follow up guide discussed at the beginning of this chapter). The detector measures the intensity of the light as the interferometric objective is actuated in the vertical direction ( $\mathrm{z}$ axis) and finds the interference maximum.

As the objective lens is moved a change of intensity due to interference will be observed for each pixel when the distance from the sample to the beam splitter is the same as the distance from the reference mirror to the beamsplitter. If the objective is moved downwards (in fig. 4) the highest points on the surface will cause interference first. This information can be used to build up a three dimensional map of the surface. Fig.4 (right) shows how the interference is built up at each pixel in the camera array.

The localisation of interference fringes during a scan of optical path length provides a way of determining a surface topography map.

The Coherence scanning interferometry used a Mirau interferometer objective lens. The basic building blocks are a microscope objective lens, a reference surface and one semi-transparent optic (often called a beamsplitter). These are configured as shown in the fig.5. There are two paths from the light source to the detector. One beam reflects off the beamsplitter, travels to the reference mirror and then reflects back, travels through the beamsplitter, to the microscope objective lens and on to the detector. The other beam travels through the beam splitter, to the test surface, reflects back to the semi-transparent mirror and then reflects from the semi-transparent mirror into the microscope objective lens and then the detector.

Areal surface texture measurement produces a three dimensional representation of a surface. The height data is represented as a height function in a plane, $\mathrm{z}(\mathrm{x}, \mathrm{y})$. Fig.6 shows a 3D topography of the final design of our pyramid sample. Three dimensional techniques give a better understanding of the surface in its functional state, and fig.7 shows an imaging stereomicroscopy (Zeiss stereo discovery V12) of pyramid structures etched on silicon substrate. The distance between each other is $136 \mu \mathrm{m}$ and the dimension of each pyramid is $40 \mu \mathrm{m} \times 40 \mu \mathrm{m}$.

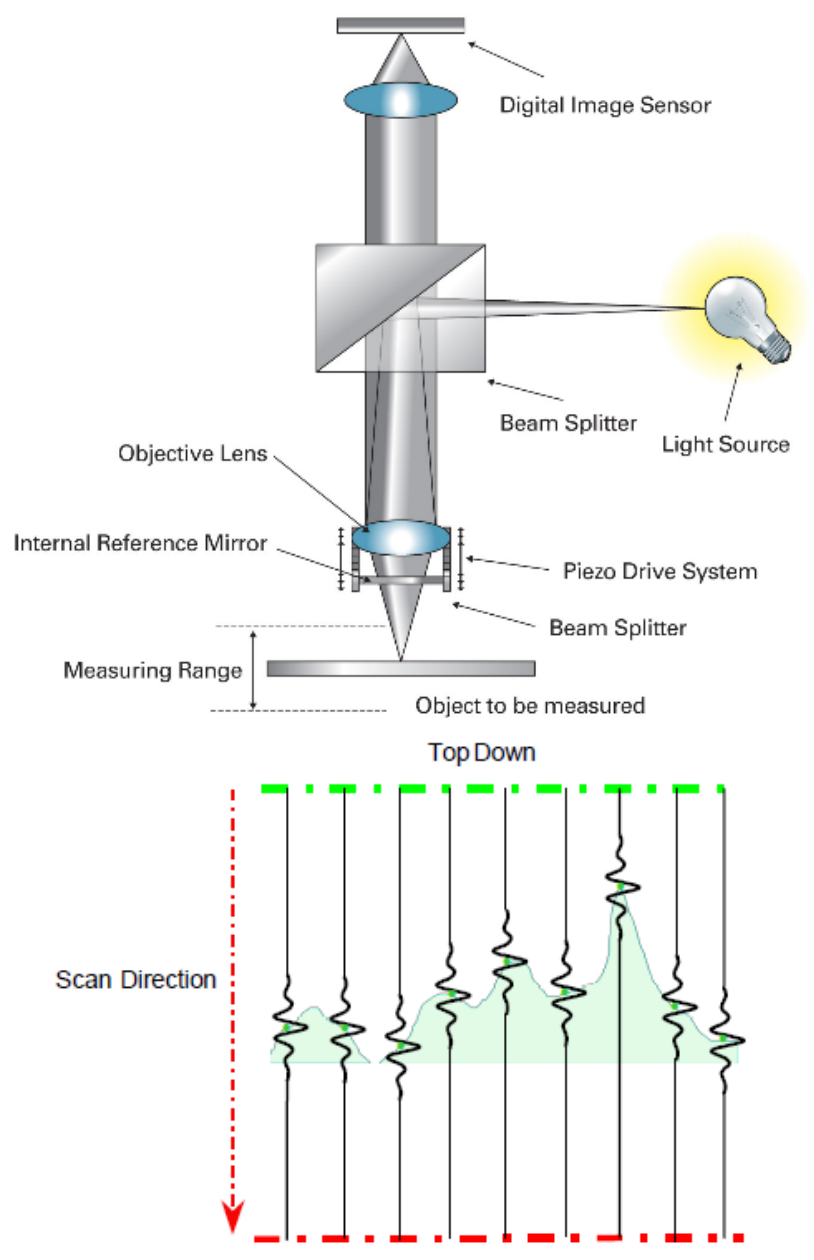

Figure 4. (Left) A typical Coherence scanning interferometry design. (right) Schematic of how to build up an interferogram on a surface using CSI. The vertical lines are intensity profiles at the image sensor

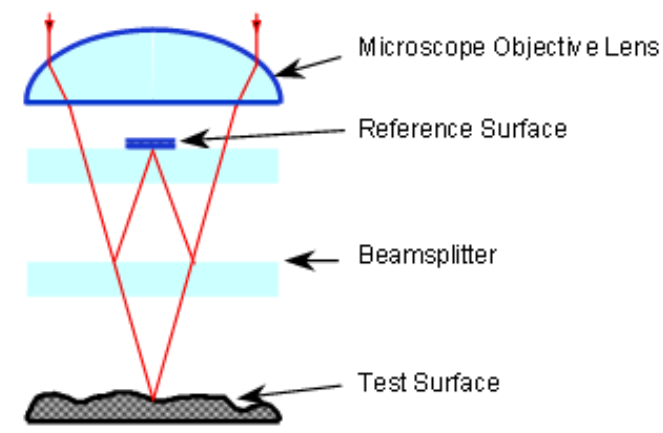

Figure 5. Schematic of a Mirau interferometer 


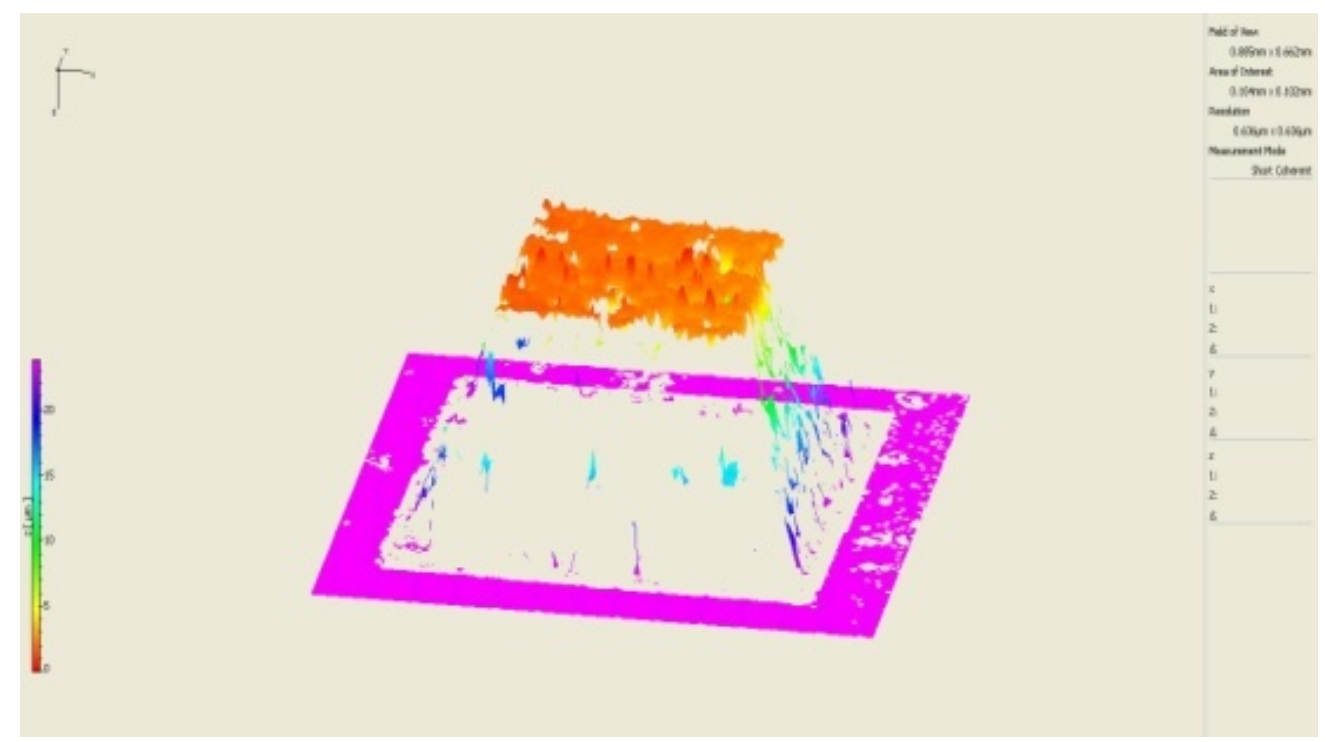

Figure 6. 3D topography of the final design of sample measured by coherence scanning interferometry Polytec MSA-500

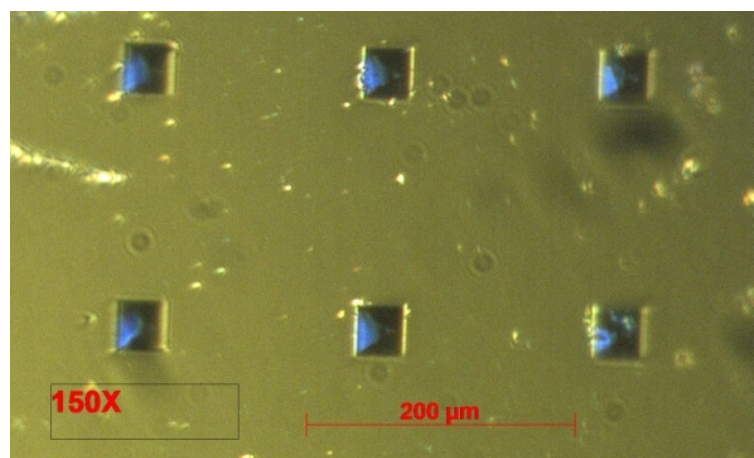

Figure 7. Imaging microscopy of the structured surface of the bioinspired sample

The root mean square value of the ordinate values within a sampling area, Sq.

The Sq parameter is defined as the root mean square value of the surface departures, $\mathrm{z}(\mathrm{x}, \mathrm{y})$, within the sampling area, thus

$$
S_{q}=\sqrt{\frac{1}{A} \iint_{A} z^{2}(x, y) d x d y}
$$

Where $\mathrm{A}$ is the sampling area, $\mathrm{xy}$. Note that equation 2 is for a continuous $\mathrm{z}(\mathrm{x}, \mathrm{y})$ function. However, when making surface texture measurements using coherence scanning interferometry (or indeed any surface texture measuring instrument), $\mathrm{z}(\mathrm{x}, \mathrm{y})$ will be determined over a discreet number of measurement points. In this case, the root mean square would be written as

$$
S_{q}=\sqrt{\frac{1}{N} \frac{1}{M} \sum_{i=1}^{N} \sum_{j=1}^{M} z_{i j}^{2}}
$$

where $\mathrm{N}$ is the number of points in the $\mathrm{x}$ direction and $\mathrm{M}$ is the number of points in the $y$ direction. The equations for the other parameters below that involve an integral notation can be converted to a summation notation in a similar manner.
The Sq parameter is the most common parameter that is used to characterise optical surfaces as it can be related to the way that light scatters from a surface.

\subsection{Optical Characterization}

The scatterometer we used in this work visualize the scattering patterns of small objects. The scatterometer measurements were helpful to understand the light scattering characteristics of pyramidal sample, and hence provide insight into sample coloration and its biological function. The power of the instrument is that it visualizes the near-field properties of the sample (with camera C1) (Fig.8), and simultaneously visualizes the far-field, hemispherical scattering properties of the study object (with camera $\mathrm{C} 2$ ). The imaging scatterometer consists of standard optical elements, except the ellipsoidal mirror. Mirror ellipsoid that it compresses a hemispherical space in a small cone, which then allows projection by common lenses. The system has an essential and sensitive point in that. The scattering of the sample must be exactly placed in the first focal point of the ellipsoid. In this have a practice is realized by observing the sample with the binocular viewer in which camera $\mathrm{C} 1$ is connected (bound), and besides by verifying that all the reflected light beams (shelves) cross (spend) a narrow diaphragm surrounding the second focal point, F2 (fig.8).

The primary beam, delivered by light source S1 and diaphragm D1, is focused by lenses L1 and L2, via a central hole in the ellipsoidal reflector, $\mathrm{M}$, on the sample, positioned in the first focal point of the reflector, F1. The positioning of the sample is visually controlled with the epi-illumination microscope consisting of lenses L2 and L3 to which camera $\mathrm{C} 1$ is connected. The beam aperture is determined by diaphragm D2. The secondary beam, delivered by light source S2, via diaphragms D3-5 and lenses L4 and L5, is focused via a beam splitter, $\mathrm{H}$, and the ellipsoidal reflector at the sample. Light scattered by the sample is focused at the second focal point of the reflector, F2, which coincides with 
the front focal point of a large-aperture photographical lens,

L6. The far-field scattering pattern, projected in plane I, is imaged by lens L7 at a digital camera, C2 . A reflected light ray, having an angle $\theta$ with the axis, leaves the second focal point with an angle $\alpha$, has a distance $r$ to the axis in the back focal plane of lens L6, and is projected at the camera chip at a distance $\mathrm{p}$ from the axis. A spatial filter in plane I blocks the zeroth-order transmitted light.

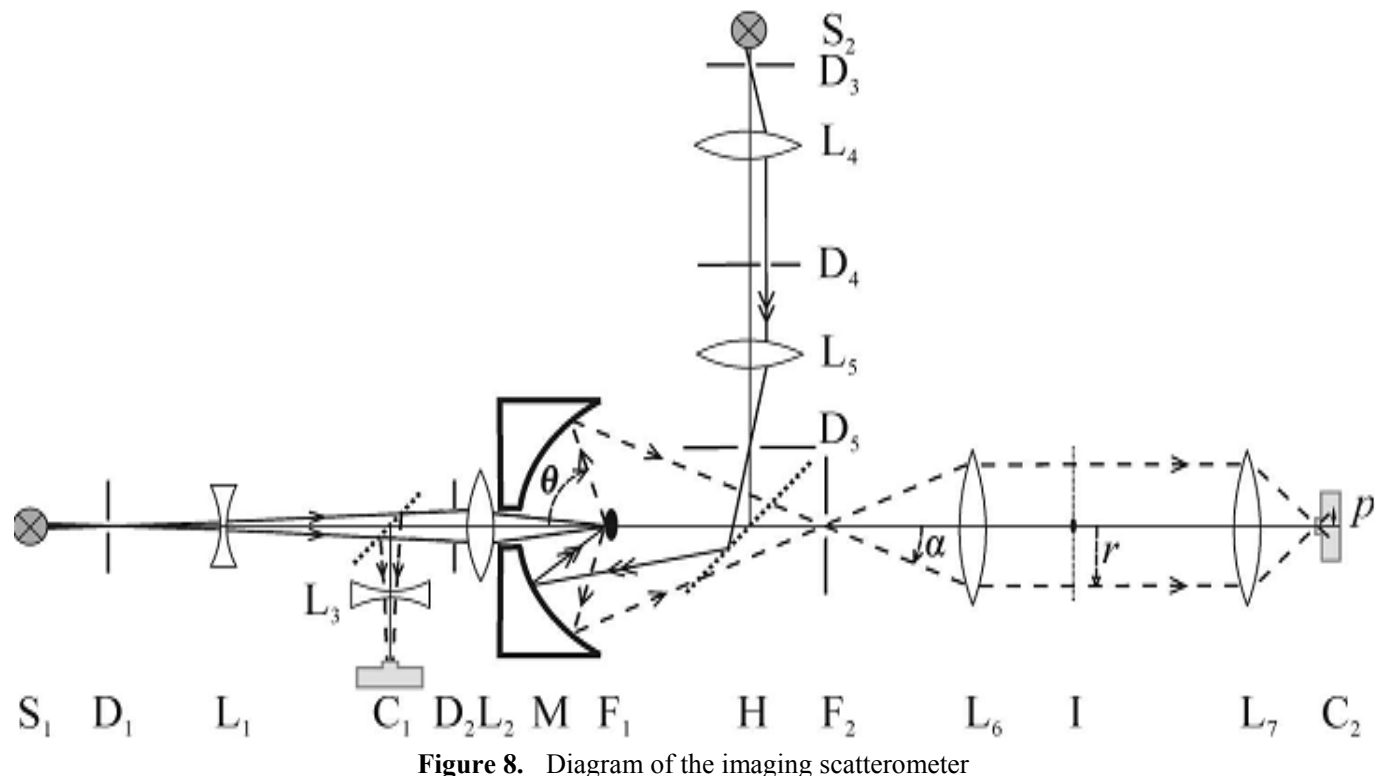

\section{Results}

\subsection{Imaging Scatterometry}

The power of the imaging scatterometer was investigated on a small fragment of a highly iridescent bioinspired sample. The primary beam with aperture $5^{\circ}$ was focused first on a flat surface of the sample and then on one of the pyramids, resulting in a $40 \mu \mathrm{m}$ diameter spot (Fig.9) The near-field photograph shows the illumination of a small area, diameter about 40 mm, with the primary beam.

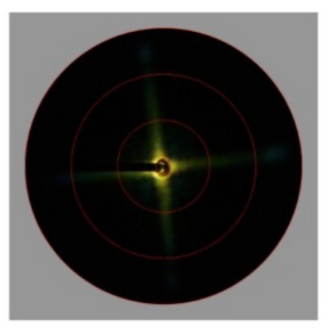

Figure 9. Imaging scatterometry of flat surface of the sample. A small fragment was positioned in the scatterometer and the primary beam was projected as a $40 \mu \mathrm{m}$ spot at a single scale

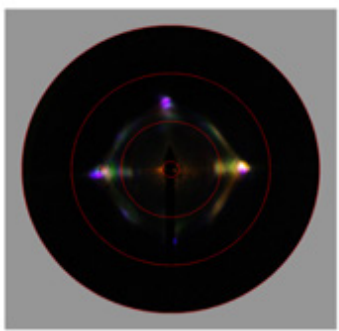

(a)

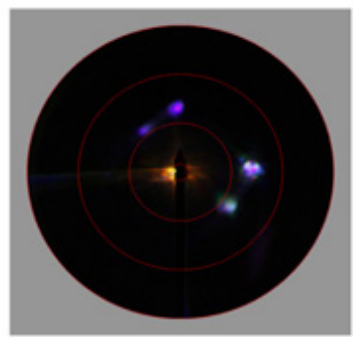

(b)

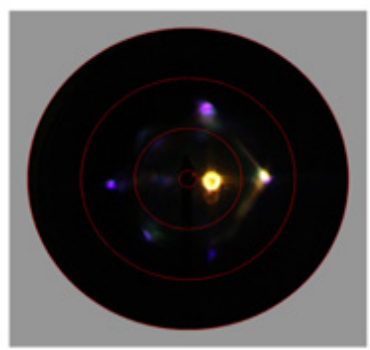

(c)

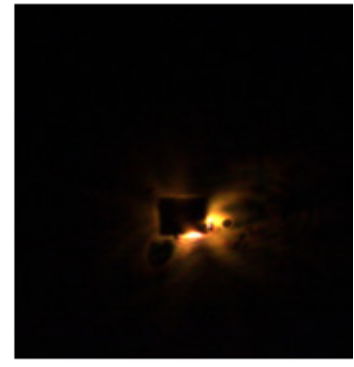

(d)

Figure 11. Imaging scatterometry of one pyramid pattern a) Wide-aperture illumination yields scattering patterns with vertically linearly polarized Light b) Rotating the sample piece over angle of $5^{\circ} \mathrm{C}$ ) Side illumination of a pyramid with a primary beam d) Wide-aperture side illumination yields scattering patterns 
The imaging scatterometer allows straightforward measurement of reflectance spectra. The incident light was scattered into a very wide angle (Fig.10).

Applying monochromatic light and using the reflection from a mirror as reference, the reflectance was calculated by integrating the reflection from spatial areas bounded by cones with apertures $30^{\circ}, 60^{\circ}$, and $90^{\circ}$.

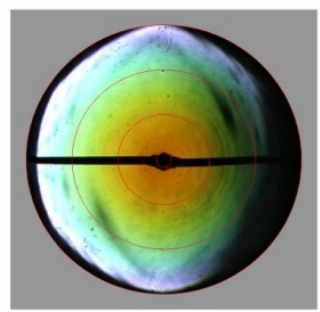

Figure 10. Wide-aperture illumination yields scattering patterns that are orange in the centre and blue at large angles. The wider secondary light beam caused the additional scattering of the scale assembly

The resulting scattering patterns, documented by the far-field camera C2 (Fig.8) are shown in (Fig.11a). The scattering patterns were directionally very restricted spots, centred around the directional angle $45^{\circ}$. These blue-purple spots from the walls of pyramid were due to the double reflection. The scattering of the illuminated pyramidal piece was investigated in another case where the angle of incidence was $5^{\circ}$. This was realized by rotating the sample around an axis perpendicular to the direction of illumination in $5^{\circ}$. The resulting scattering patterns, documented by the far-field camera C2 (Fig 8) are shown in (Fig.11b).

\subsection{Detailed Modelling}

The reflectance of the multilayer in our model was calculated by applying the classical multilayer theory for dielectric media

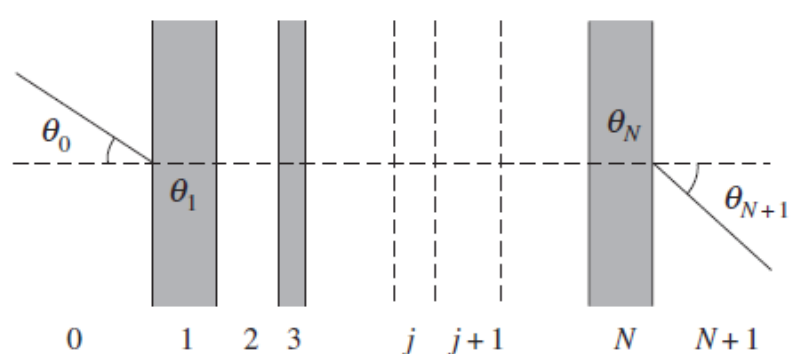

Figure 12. Diagram of light propagation through a multilayer existing of $\mathrm{N}$ layers with refractive indices $\mathrm{nj},=1,2, \ldots, \mathrm{N}$, bordered by media with refractive index $\mathrm{n} 0$ and $\mathrm{nN}+1$. The angles of light incidence and refraction at the interface of media $\mathrm{j}$ and $\mathrm{j}+1$ are $\theta_{j}$ and $\theta_{j+1}$, respectively

This approach is based on the linearity of the equation for propagation of light. All the layers are assumed to be isotropic and homogenous. Let us consider a system of the $\mathrm{N}$ parallel layers placed between two semi-infinite media (Fig. 12). Let all the media to be linear homogeneous and isotropic. We have to find now a suitable mathematical approach for determination of the reflection and transmission coefficients of light propagated through the layered structure at the defined wavelength $\lambda$, angle of incidence $\theta_{0}$, thicknesses of the layers $d_{j}(j=1,2, \ldots N)$, their refractive indexes and extinction coefficients $\tilde{n}=n_{j}-i k_{j}$; the imaginary part of the refractive index, $k_{j}$, is related to the absorption coefficient of the medium, $\alpha_{j}$, by $k_{j}=\alpha_{j} \lambda / 4 \pi$, where $\lambda$ is the light wavelength. The propagation of light through this multilayer is governed by Snell's Law:

$$
\tilde{n}_{j} \sin \theta_{j}=n_{0} \sin \theta_{0}, \quad \mathrm{j}=1,2, \ldots, \mathrm{N}+1
$$

Where the angle of incident $\theta_{j}$ of the light ray at the interface of media $j$ and $j+1$ can be complex. The light propagation through the multilayer is described by the transfer matrix

$$
\begin{aligned}
M & =\left(\begin{array}{ll}
M_{11} & M_{12} \\
M_{21} & M_{22}
\end{array}\right) \\
& =D_{0}^{-1}\left[\prod_{j=1}^{N} D_{j} P_{j} D_{j}^{-1}\right] D_{N+1}
\end{aligned}
$$

Where

$$
D_{j}=\left(\begin{array}{cc}
p_{j} & p_{j} \\
q_{j} & -q_{j}
\end{array}\right), j=0,1,2, \ldots . ., N+
$$

and

$$
P_{j}=\left(\begin{array}{cc}
s_{j} & 0 \\
0 & 1 / s_{j}
\end{array}\right), j=1,2, \ldots . ., N,
$$

with $\mathrm{pj}=1$ and $q_{j}=\widetilde{n}_{j} \cos \theta_{j}$ for $\mathrm{TE}$ waves, $p_{j}=\cos \theta_{j}$ and $q_{j}=\tilde{n}_{j}$ for TM waves and

sj $=\exp \left(i \varphi_{j}\right)$ with $\varphi_{j}=2 / \lambda \tilde{n}_{j} d_{j} \cos \theta_{j}$. The reflectance of the multilayer then is

$$
R=\left|\frac{M_{21}}{M_{11}}\right|^{2}
$$

and the transmittance is

$$
T=\frac{n_{N+1}}{n_{0}} \frac{\cos \theta_{N+1}}{\cos \theta_{0}}\left|\frac{1}{M_{11}}\right|^{2}
$$

The computational time needed to evaluate the matrix elements of equation (Equation 4) can be substantially reduced by realizing that in equation 4

$$
D_{j} P_{j} D_{j}^{-1}=\left(\begin{array}{cc}
a_{j} & i b_{j} p_{j} / q_{j} \\
i b_{j} p_{j} / q_{j} & a_{j}
\end{array}\right)
$$

where $a_{j}=\cos \varphi_{j}$ and $b_{j}=\sin \varphi_{j}$ 
To simulate the spectral response according to the wavelength, we modelled a pattern of pyramid with the same coating experimentally deposited, and by paying attention to the behavior of the variation of coating according to the angle of surface. Then, we simulated a white lighting to have the spectral response of the illuminated coating, and we observed the reflections when we send a package of the beams of light with the same power.

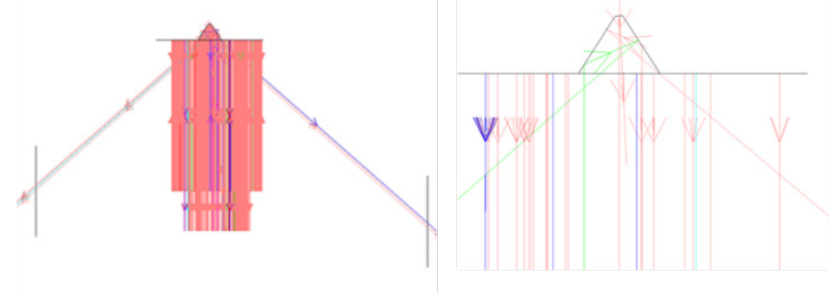

Figure 13. Modelisation of a pyramid pattern

For each specular reflection, we calculated the reflected intensity and then we cumulated the sum of the powers of the rays which reached three detectors (one frontal and two side detectors) and we repeated it for various wavelengths. For the side detectors, the result of simulation is a rear-view mirror reflexion of blue colour. (see fig.14 b).

In the case of the detector in straight line, the dominant wavelength at normal incidence, at the maximum of the reflectance spectrum, is found to be $540 \mathrm{~nm}$ which, according to a standard colorimetric classification [20] (fig.14.a), corresponds to a "green" perception. We can also observe a second peak less intense at $670 \mathrm{~nm}$. The coloration of the sample is due to the contribution of these two peaks. This spectrum is consistent with the perception. This is what is observed by naked eye, orange colour or orange-green, when viewing the sample under normal incidence. We found also the same colour in the center of the wide-aperture illumination yields scattering patterns (fig.10).

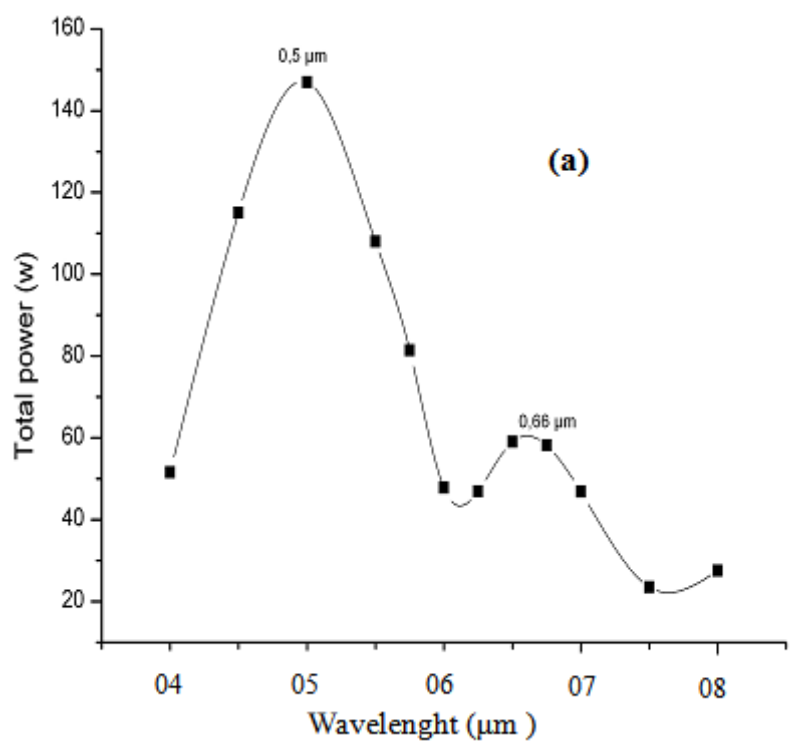

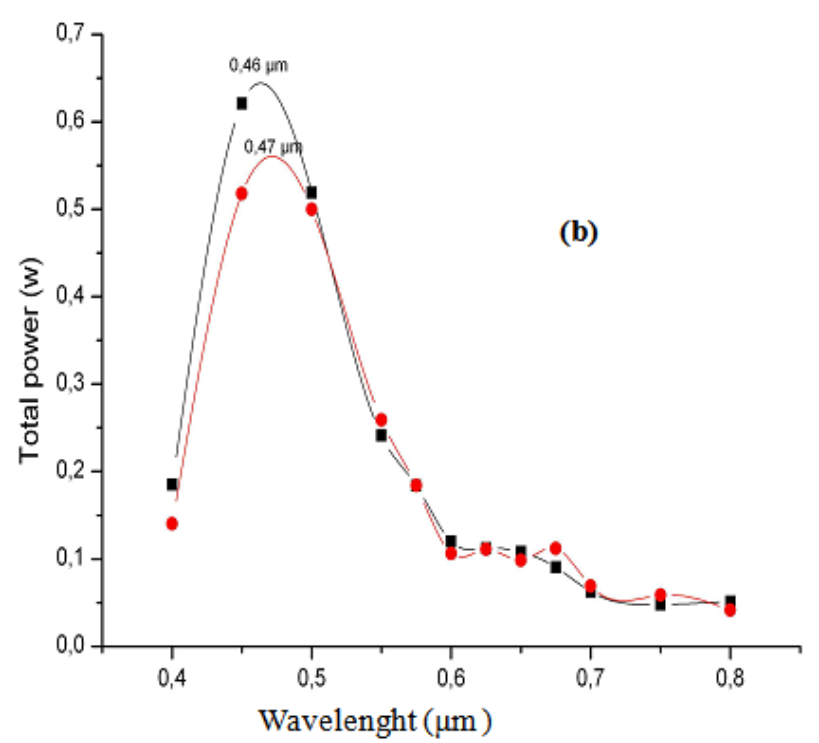

Figure 14. Predicted reflectance of the bioinspired structure. a) with frontal detector b) with lateral detectors; red spectrum for the left detector and black spectrum for the right one

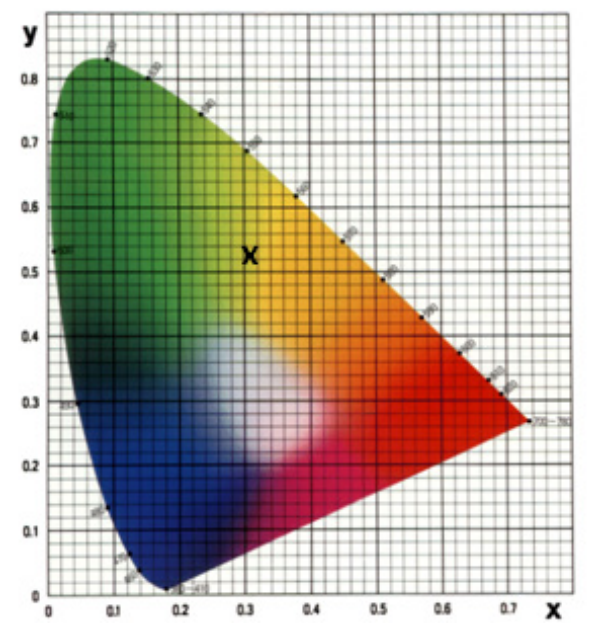

Figure 15. Predicted colour of the bioinspired structurefrom the chromaticity diagrams

In the case of the detector in a straight line, we can guess the orange colour or orange-green colour from the reflectance spectra (fig.10.a) and we found the same colour in the center of the wide-aperture illumination yields scattering patterns (fig.7).

The coloration produced by the pyramid structure in reflection under a specified illumination can be quantified in terms of chromaticity coordinates $(\mathrm{X}, \mathrm{Y}, \mathrm{Z})$ [13-16]. The latter were calculated from the reflectance spectra $R(\lambda)$ using:

$$
\begin{aligned}
& X=k \int R(\lambda) L(\lambda) x(\lambda) d \lambda \\
& Y=k \int R(\lambda) L(\lambda) y(\lambda) d \lambda \\
& Z=k \int R(\lambda) L(\lambda) z(\lambda) d \lambda
\end{aligned}
$$


Where $L(\lambda)$ is the source spectrum, $x(\lambda), y(\lambda)$ and $z(\lambda)$ are the CIE 1931 colour-matching functions, and $\mathrm{k}$ is a normalization constant so that $\mathrm{X}+\mathrm{Y}+\mathrm{Z}=1$. The coordinates $(X, Y)$ which define the chromaticity of the colour in a so-called chromaticity diagram, describe the same colour as we have seen previously in the reflectance spectra (Fig.14a) and the center of the wide-aperture illumination yields scattering patterns (Fig.10).

\section{Conclusions}

The knowledge acquired in performing this study has been used to develop an artificial structured surface which reproduces the visual effect found with the Chrysochroa vittata beetle. In this insect, a combination of multilayer interference, optical gratings, photonic crystals and other optical structures gives rise to complex colour mixing. Although the physics of structural colours is well understood, it remains a challenge to create artificial replicas of natural photonic structures. O. Deparis et al. [25] and J.P. Vigneron et al. [1] used to develop also an artificial bio-inspired multilayer system which reproduces the visual effects provided by the insects cuticle. But our main goal in this work, is to analyze the role of roughness. We determined the optical properties changing of these structured surfaces based on the characteristics of surface roughness. We used a combination of pholithographic process, anisotropic wet etching, to fabricate pyramid structures on silicon subsrate, and we exploited the robustness of this thin-layer design, the changing colours of Chrysochora vittata were copied into a bio-inspired artificial reflector, with thin metal layers acting as periodic perturbation of a dielectric. Morphology characterization is used in this paper thanks to coherence scanning interferometry and stereomicroscopy and we measured the optical properties thanks to scatterometry technics. The measurements compared to theoretical modelling and numerical simulations will confirm the natural-artificial similarities.

In this paper, we operated the strength of the surface roughness made by micro and nanofabrication to create the multiple reflections due to the internal surfaces of the pyramid to mimic or to produce samples based on natural structures where optical properties can be interesting. These Iridescent bio-inspired fabrications could find many uses in the hands of objects designers.

\section{ACKNOWLEDGMENTS}

This investigation was conducted with the support of Communauté Française de Belgique through ARC project 10/15-033 "Propriétés optiques des structures naturelles et artificielles».

We thank Prof. Doekele G. Stavenga for the scattering measures performed within the University of Groningen, Department of Applied Physics.

\section{REFERENCES}

[1] J.-P. Vigneron, M. Rassart, C. Vandenbem, V. Lousse, O. Deparis, L.P. Biro, D. Dedouaire, A. Cornet, P. Defrance, «Spectral filtring of visible light by the cuticle of metallic woodboring beetles and microfabrication of a matching bioinspired material» Phys. Rev. E, (2005).

[2] Lord Rayleigh, O.M., F.R.S., Phil. Mag. 37, 98 (1918).

[3] Lord Rayleigh, F.R.S., Roy. Soc. Proc. A 103, 233 (1923).

[4] K. Gentil, Z. Morph. Okol. Tiere 38, 344 (1942).

[5] C. G. Bernhard, G. Gemne, and A. R. Moeller, Quart. Rev. Biophys. 1, 89 (1942).

[6] J. Zi, X. Yu, Y. Li, X. Hu, C. Xu, X. Wang, X. Liu, and R. Fu, Proc. Natl. Acad. Sci. USA 100, 12576 (2003).

[7] A. R. Parker, V. L. Welch, D. Driver, and N. Martini, Nature 426, 786 (2003).

[8] J. P. Vigneron, J.-F. Colomer, N. Vigneron, and V. Lousse, Phys. Rev. E 72, 061904 (2005).

[9] Durrer, H. 1977 Schillerfarben der Vogelfeder als Evolutionsproblem. Denkschr. Schweiz. Naturforsch. Ges. 91, $1-126$.

[10] Kinoshita, S. 2008 Structural colors in the realm of nature. Singapore: World Scientific.

[11] Land, M. F. 1972 The physics and biology of animal reflectors. Prog. Biophys. 24, 77-105. (doi:10.1016/00796107(72) 90004-1)

[12] Huxley, J. The coloration of Papilio zalmoxis and P. antimachus and the discovery of Tyndall blue in butterflies, Proc. R. Soc. Lond. B 193 pp441-453 (1976)

[13] R. C. Jaeger, Introduction To Microelectronic Fabrication, 2 ed. Auburn, Upper Saddle River - Prentice Hall, 2002.

[14] M. J. Madou, Fundamentals of Microfabrication: The Science of Miniaturization, 2 ed.: CRC Press, 2002.

[15] V. J. Logeeswaran, et al., "Harvesting and Transferring Vertical Pillar Arrays of Single-Crystal Semiconductor Devices to Arbitrary Substrates," Electron Devices, IEEE Transactions on, vol. 57, pp. 1856-1864, 2010.

[16] Chen, et al., Effect of process parameters on the surface morphology and mechanical performance of silicon structures after deep reactive ion etching (DRIE) vol. 11. New York, NY, ETATS-UNIS: Institute of Electrical and Electronics Engineers, 2002.

[17] K. R. Williams, R. S. Muller, "Etch rates for micromachining processing," In: IEEE J. Microelectromechan. Syst. 1996, 5, 256-269; K. R. Williams II, K. Gupta, M. Wasilik, "Etch rates for micromachining processing - Part II." IEEE J. Microelectromechan. Syst. 2003, 12, 761- 778.

[18] Swanepoel, R. (1985). Determining refractive index and thickness of thin films from wavelength measurements only. JOSA A, 2(8), 1339-1343.

[19] Manifacier, J. C., Gasiot, J., \& Fillard, J. P. (1976). A simple method for the determination of the optical constants $n, k$ and the thickness of a weakly absorbing thin film. Journal of Physics E: Scientific Instruments, 9(11), 1002. 
[20] R. S. Berns, Billmeyer and Saltzman's Principles of Color Technology, 3rd Edition (Wiley-Interscience, New York, 2000), ISBN 047119459X.

[21] D. G. Stavenga, M. A. Giraldo, and B. J. Hoenders, "Reflectance and transmittance of light scattering scales stacked on the wings of pierid butterflies," Opt. Express 14, 4880-4890 (2006).

[22] Serge Berthier, Les couleurs des Papillons, ou l'impérative beauté: propriétés optiques des ailes de papillons (Springer Verlag, Paris, 2000).
[23] J.P. Vigneron, J.-F. Colomer, N. Vigneron and V. Lousse, "Natural layer-by-layer photonic structure in the squamae of Hoplia coerulea (Coleoptera)," Phys. Rev. E 72, 061904 (2005).

[24] B. Gralak, G. Tayeb, S. Enoch, "Morpho butterflies wings color modeled with lamellar grating theory," Opt. Express 9, 567 (2001).

[25] Deparis, O. , Vandenbem, C. , Welch, V. , Rassart, M. , Lousse, V. , Vigneron, J-P. , De Vriendt, V. \& Lucas, S. 1 janv. 2007 Conference on Lasers and Electro-Optics Europe Technical Digest. 